\title{
Evolution of microflares associated with bright points in coronal holes and in quiet regions
}

\author{
S. Kamio, W. Curdt, L. Teriaca, and D. E. Innes \\ Max-Planck-Institut für Sonnensystemforschung (MPS), Max-Planck-Str. 2, 37191 Katlenburg-Lindau, Germany \\ e-mail: skamio@spd.aas.org
}

Received 8 September 2010 / Accepted 22 February 2011

\section{ABSTRACT}

\begin{abstract}
Aims. We aim to find similarities and differences between microflares at coronal bright points found in quiet regions and coronal holes, and to study their relationship with large scale flares.

Methods. Coronal bright points in quiet regions and in coronal holes were observed with Hinode/EIS using the same sequence. Microflares associated with bright points are identified from the X-ray lightcurve. The temporal variation of physical properties was traced in the course of microflares.

Results. The lightcurves of microflares indicated an impulsive peak at hot emission followed by an enhancement at cool emission, which is compatible with the cooling model of flare loops. The density was found to increase at the rise of the impulsive peak, supporting chromospheric evaporation models. A notable difference is found in the surroundings of microflares; diffuse coronal jets are produced above microflares in coronal holes while coronal dimmings are formed in quiet regions.

Conclusions. The microflares associated with bright points share common characteristics to active region flares. The difference in the surroundings of microflares are caused by open and closed configurations of the pre-existing magnetic field.
\end{abstract}

Key words. Sun: corona - Sun: coronal mass ejections (CMEs) - Sun: UV radiation

\section{Introduction}

Coronal bright points are small roundish or loop-shaped features seen in X-ray and UV emissions all over the Sun. Their typical size is $5-10^{\prime \prime}$, and they last for several hours or longer (Golub et al. 1974). Thanks to the high sensitivity of the X-ray telescope (XRT; Golub et al. 2007) on Hinode (Kosugi et al. 2007a), bright points are found to be highly dynamic and time varying (Cirtain et al. 2007; Kotoku et al. 2007b). It is known that bright points occasionally undergo a sudden increase in emissions on a time scale of several minutes (Strong et al. 1992; Koutchmy et al. 1997). In this paper, we call these transient brightenings as in the corona microflares, and focus on the microflares associated with bright points in quiet regions and coronal holes.

The study of microflares is important for understanding the process of energy release in the corona, since they ubiquitously occur in quiet regions and coronal holes. Although the size of microflares is much smaller than that of large flares in active regions, they share some common characteristics. Bright points are formed above small magnetic concentrations in the photosphere (Golub et al. 1977), while active regions are a manifestation of large scale magnetic flux. Recent observations have revealed small scale ejections which resemble coronal mass ejections (CMEs) from active regions (Innes et al. 2009, 2010). It is worthwhile to study the evolution of microflares and examine if their properties agree with the standard flare model (Carmichael 1964; Sturrock 1966; Hirayama 1974; Kopp \& Pneuman 1976). It is essential to trace the physical properties such as temperature and density in the course of microflares in order to compare microflares with the flare model.

A sequence of XRT images show that many jets originate from microflaring bright points in coronal holes, while coronal jets are not common to bright points in quiet regions. One question to be answered is whether these two types of microflares are caused by the same mechanism or not. The distinct difference gives insights into interactions between microflares and magnetic fields in the surroundings. Jets in coronal holes have been extensively studied (Savcheva et al. 2007; Shimojo et al. 2007; Subramanian et al. 2010). They are clearly observed against the dark background in coronal holes. However, a detailed comparison of microflares in quiet regions and coronal holes has not been made yet.

In this respect, we carried out observations to trace the temporal evolution of microflares at bright points. Determining physical properties of microflares is of great importance. To compare bright points in a quiet region and in a coronal hole, both targets were monitored with the same observing sequence near the solar limb. The paper is organized as follows; the methodology is explained in Sect. 2; the observations and data reduction procedures are described in Sect. 3; the properties of microflares in a quiet region and in a coronal hole are presented in Sect. 4. the formation of microflares and their similarity to active region flares are discussed in Sect. 5; and the conclusions are summarized in Sect. 6.

\section{Methodology}

The primary purpose of our study is to find similarities and differences in the evolution of microflares in quiet regions and in coronal holes. To this end, the same observing sequence was carried out at four locations near the limb: quiet regions in the east and in the west and polar coronal holes at the north and at the south. 

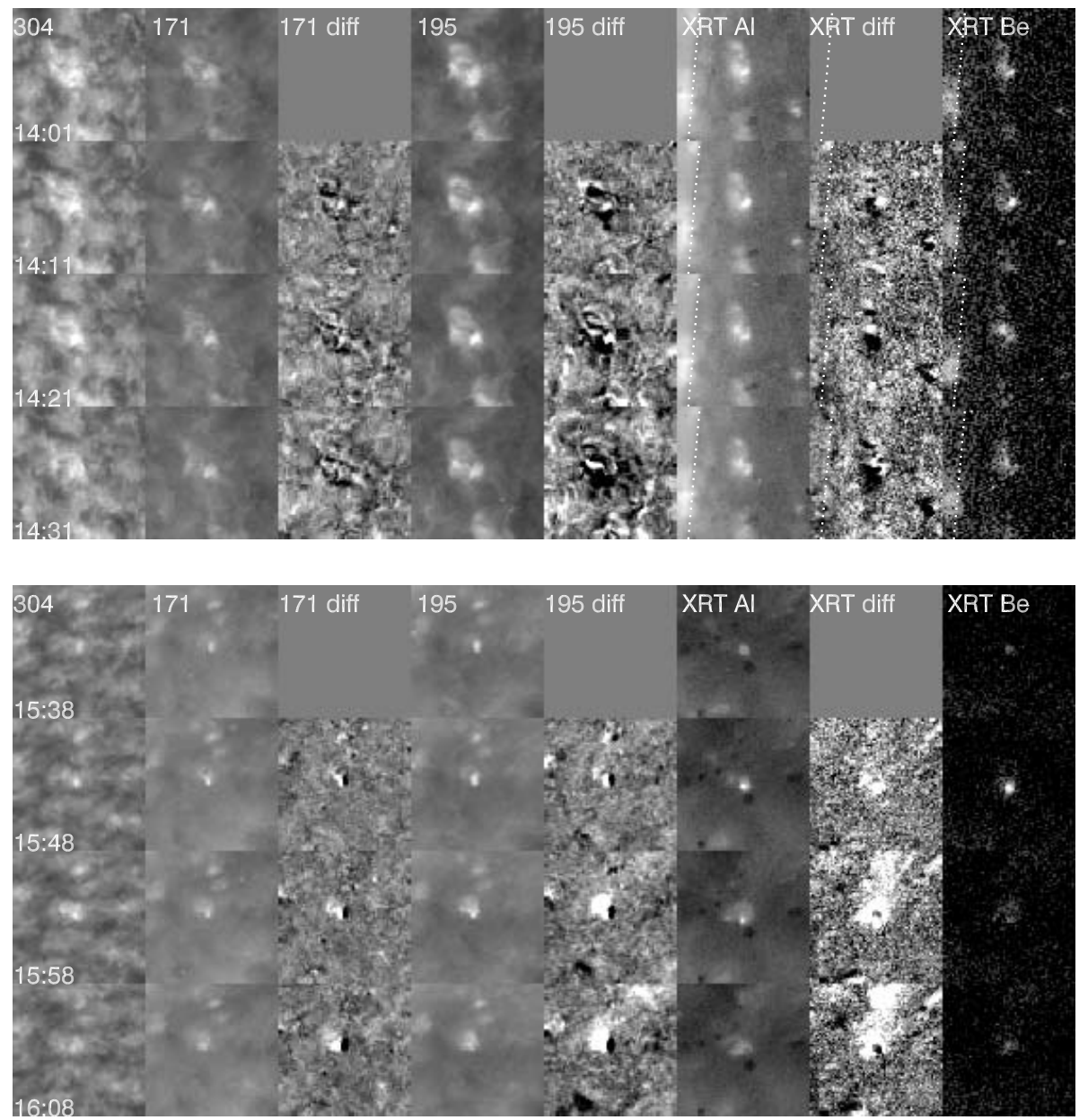

Fig. 1. Top: temporal evolution of a bright point in a quiet region (QR1). Columns display time series of SECCHI/EUVI images in $\lambda 30.4 \mathrm{~nm}$ (He II), $\lambda 17.1 \mathrm{~nm}(\mathrm{Fe}$ Ix/x), and $\lambda 19.5 \mathrm{~nm}$ (Fe xII) and XRT images with Al_poly and Be_thin filters with 10 min intervals. Difference images are indicated by "diff" on columns. Dimension of the each panel is $100^{\prime \prime} \times 100^{\prime}$. Dotted lines on the XRT images mark the solar limb. Bottom: temporal evolution of a bright point in a coronal hole $(\mathrm{CH} 1)$.

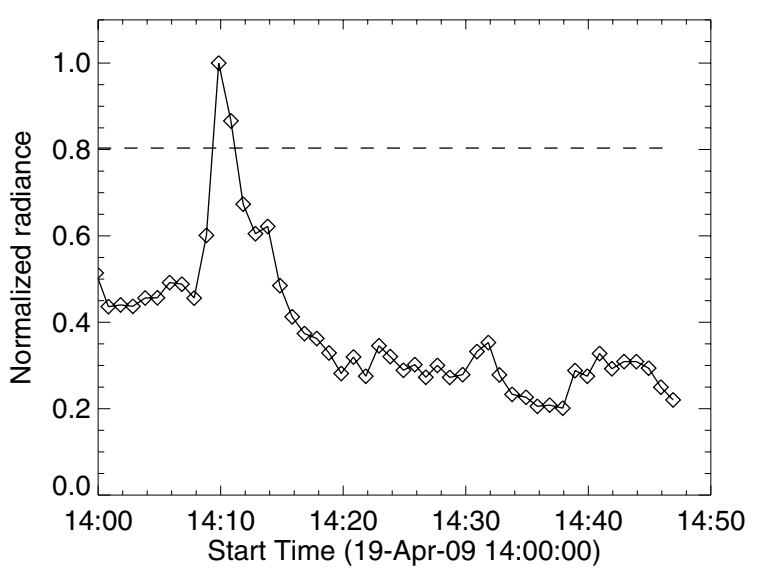

Fig. 2. An X-ray lightcurve of a bright point indicating a microflare at 14:10 UTC. Dashed line marks $3 \sigma$ level of the light curve.

We use coronal imagers to trace the evolution of microflares and coronal structures in the surroundings. A sequence of XRT images was employed to identify microflares at coronal bright points. Since the X-ray images were recorded at 1 min cadence, they had the best temporal coverage of the evolution. An X-ray lightcurve of a bright point is created by spatially integrating
X-ray flux over the bright point. We selected impulsive enhancements exceeding the $3 \sigma$ level of the lightcurve, where $\sigma$ is the standard deviation. Figure 2 shows an X-ray lightcurve of a bright point. A peak at 14:10 UTC exceeding $3 \sigma$ level is selected as a microflare (QR1 in Table 2), and the peak time is defined as the time of the microflare. A $4^{\prime \prime} \times 2^{\prime \prime}$ summing was applied to the XRT data in order to match the EIS resolution. The brightest pixel in the summed XRT data at the time of the microflare is selected for following analysis as it represents the microflare.

In addition, time series of EUV images obtained by SECCHI/EUVI (Howard et al. 2008; Wülser et al. 2004) on STEREO (Kaiser et al. 2008) are analyzed to study the coronal structures associated with these microflares. Difference images are created to detect small changes in emission, which is a widely used technique since the discovery of coronal dimmings (Thompson et al. 1998).

At the same time, physical properties of microflares are deduced. The EUV Imaging Spectrometer (EIS; Culhane et al. 2007a) on Hinode carried out repeated fast raster scans to study the evolution of microflares at different temperatures ranging from the transition region to the corona. Although the temporal resolution of the data is not good as compared to XRT, the spectra allow us to deduce the temperature evolution of the microflares. The observed time difference between the hot and cool emission lines is compared with a simple loop cooling model 
Table 1. List of emission lines observed with EIS.

\begin{tabular}{lcc}
\hline \hline Ion & $\lambda / \mathrm{nm}$ & $\log T_{\mathrm{e}} / \mathrm{K}$ \\
\hline Fe VIII & 18.660 & 5.6 \\
Fe XII & 18.688 & 6.1 \\
Fe XI & 19.281 & 6.1 \\
O v & 19.291 & 5.4 \\
Fe XII & 19.512 & 6.1 \\
Fe XVII & 25.487 & 6.6 \\
He II & 25.632 & 4.7 \\
Si x & 26.106 & 6.1 \\
Fe xv & 28.416 & 6.3 \\
\hline
\end{tabular}

by Cargill et al. (1995), by analogy with large scale flares. In addition, the line ratio technique was applied to detect density enhancements in the corona, which we expected from chromospheric evaporation in the standard flare model. Our method is to identify a distinct microflare and to track the temporal evolution, hence the number of events detected in our study is not sufficient for a statistical study.

\section{Observations and data reduction}

Four sets of coordinated observations were carried out on 19 and 21 April 2009. No active regions appeared at that time. The observing sequence is composed of rapid raster scans by EIS and time series of coronal images for three hours in each location.

EIS on Hinode repeated sparse rasters at 6 min cadence. Spectra of emission lines listed in Table 1 were recorded with the $2^{\prime \prime}$ wide slit and at exposure time of $25 \mathrm{~s}$. Emission lines were selected to cover a wide range of temperature within a limited telemetry volume. The resultant pixel resolution is $2^{\prime \prime} \times 2^{\prime \prime}$ after summing along the slit to increase the signal to noise ratio. Each raster scan consists of 12 exposures with $4^{\prime \prime}$ step size, and an area of $44^{\prime \prime} \times 320^{\prime \prime}$. The duration of the sequence was one hour due to the eclipse periods during the Hinode Sun-synchronous orbit.

EIS data are calibrated with the standard procedures provided in the Solar Software tree (SSW; Freeland \& Handy 1998). A single Gaussian fit was applied to spectra to deduce radiance, Doppler velocity, and line width. A single Gaussian results in a reasonable fit in quiet conditions. Spectral profiles taken during the microflares occasionally deviate from Gaussian profiles because of enhancements in the wings, which are presented in Sect. 4.3. As for EIS, the Doppler shift is deduced after compensating for the instrumental effect caused by temperature variations (Kamio et al. 2010b) and using the rest wavelengths of emission lines identified by Brown et al. (2008). The contribution of the Si x $\lambda 25.637 \mathrm{~nm}$ blending in the He II $\lambda 25.632 \mathrm{~nm}$ is subtracted by using the other $\mathrm{Si} x \lambda 26.106 \mathrm{~nm}$ emission (Kamio et al. 2009). Although a coronal blending remains in the red wing of the spectrum, He II is the dominant emission at that wavelength.

Time series of coronal images were obtained to study the evolution of the microflares. XRT recorded soft X-ray images with Al_poly and Be_thin filters. The former is sensitive to low temperature coronal emission down to $1 \times 10^{6} \mathrm{~K}$ and shows coronal structures in quiet regions. The latter detects the higher temperature corona and normally results in a weak signal in quiet regions. It was intended to capture the hot component in coronal bright points. A pair of X-ray filter images were obtained every $60 \mathrm{~s}$. The area of the XRT images were $384^{\prime \prime} \times 384^{\prime \prime}$, and the EIS area was covered. Co-alignment between EIS and XRT was performed by comparing the Fe XII $\lambda 19.512 \mathrm{~nm}$ radiance map from EIS and XRT Al_poly images. We estimate a co-alignment error of $4^{\prime \prime}$, which corresponds to the scan step of the EIS data. Other emission lines are registered after compensating for the north south offset of the EIS spectra (Kamio et al. 2010b).

STEREO EUVI obtained images with filters of $\lambda 17.1 \mathrm{~nm}$ (Fe IX/X), $\lambda 19.5 \mathrm{~nm}$ (Fe XII), and $\lambda 30.4 \mathrm{~nm}$ (He II). EUVI images with the set of filters were recorded at a cadence of $10 \mathrm{~min}$. A coordinate conversion is necessary to find the bright points observed with XRT in the SECCHI/EUVI images. The separation angles between the Earth and STEREO-A and B spacecrafts were $46.7^{\circ}$ and $47.0^{\circ}$, respectively.

Since the Hinode spacecraft is in low-Earth orbit (Kosugi et al. 2007a), XRT images are regarded as Earth view. Assuming that a coronal bright point was located on the solar surface, Heliocentric Earth Ecliptic (HEE) ${ }^{1}$ coordinates of the bright point are deduced from its apparent position in XRT images. The coordinates are transformed into STEREO spacecraft views by using the STEREO SPICE software package provided in the SSW tree. The position of the coronal bright point on the STEREO EUVI images is determined by taking $x$ and $y$ coordinates in the STEREO spacecraft view.

\section{Results}

In our data set, 10 microflares are identified in X-ray images (7 in quiet region and 3 in coronal hole) and are listed in Table 2. In the following, two representative events are described: one in a quiet region and the other in coronal hole. They are labelled as QR1 and CH1 in Table 2. Similarities and differences between the quiet region and the polar coronal hole and the implications for the coronal structures are discussed in Sect. 5.

\subsection{Coronal structures}

The top panel of Fig. 1 shows a time series of a bright point in a quiet region near the East limb (QR1 in Table 2). Each column presents a $100^{\prime \prime} \times 100^{\prime \prime}$ section of SECCHI/EUVI images in $\lambda 30.4 \mathrm{~nm}(\mathrm{He}$ II), $\lambda 17.1 \mathrm{~nm}(\mathrm{Fe} \mathrm{IX} / \mathrm{x})$, and $\lambda 19.5 \mathrm{~nm}$ (Fe XII), and XRT images with Al_poly and Be_thin filters. As the Al_poly images show, the bright point was located just 20" inside the limb. The top row shows pre-event images of the bright point at 14:01 UTC. Enhanced emission was detected at the center of all panels at 14:11 UTC, which suggests that the microflare took place in a wide temperature range. A significant emission increase is detected with the XRT Be_thin filter, which is rare in a quiet region. This is indicative of a hot component produced by the microflare, since the Be_thin filter is only sensitive to the high temperature corona.

Difference images in $\lambda 17.1 \mathrm{~nm}(\mathrm{Fe} \mathrm{Ix} / \mathrm{x})$, and $\lambda 19.5 \mathrm{~nm}$ (Fe XII), and XRT Al_poly are derived by subtracting pre-event images at 14:01 UTC to emphasize the temporal variation. Emissions of the microflare reached their peaks just before 14:11 UTC while a significant dimming was observed in the surrounding. A careful analysis of the high cadence (1 min) XRT time series shows that the dimming started at 14:08 UTC, when the X-ray flux of the microflare underwent a sharp rise. The dimming continued to expand until 14:31 UTC. As the STEREO-B spacecraft was separated from the Earth by $47.0^{\circ}$, SECCHI/EUVI provided a better coverage of the region close to the limb. The difference images in $\lambda 19.5 \mathrm{~nm}$ show that the size of the dimming expanded to $30^{\prime \prime}$. The dimming was also seen in $\lambda 17.1 \mathrm{~nm}$, but was restricted to $10^{\prime \prime}$, from the bright point.

\footnotetext{
1 http://secchi.nrl.navy.mil/wiki/uploads/Main/ coordinates.pdf
} 
Table 2. Properties of microflares

\begin{tabular}{|c|c|c|c|c|c|c|c|c|c|c|}
\hline \multirow{2}{*}{\multicolumn{2}{|c|}{ Location }} & \multicolumn{2}{|c|}{ Coordinates } & \multirow{2}{*}{$\begin{array}{l}\text { Diameter } \\
(\mathrm{cm})\end{array}$} & \multirow{2}{*}{$\begin{array}{l}\text { Area } \\
\left(\mathrm{cm}^{2}\right)\end{array}$} & \multirow{2}{*}{$\begin{array}{l}\text { Density } \\
\left(\mathrm{cm}^{-3}\right)\end{array}$} & \multirow{2}{*}{$\begin{array}{l}\text { Delay } \\
(\mathrm{min})\end{array}$} & \multirow{2}{*}{$\begin{array}{l}\text { Cooling time } \\
(\mathrm{min})\end{array}$} & \multirow{2}{*}{$\begin{array}{c}\text { Total EM } \\
\left(\mathrm{cm}^{-3}\right)\end{array}$} & \multirow{2}{*}{$\begin{array}{c}\text { Radiative loss rate } \\
\left(\mathrm{erg} \mathrm{s}^{-1}\right)\end{array}$} \\
\hline & & $X$ & $Y$ & & & & & & & \\
\hline \multirow{7}{*}{ QR } & 1 & $-925^{\prime \prime}$ & $115^{\prime \prime}$ & $4 \times 10^{8}$ & $1.1 \times 10^{17}$ & $4 \times 10^{9}$ & 12 & 9 & $1.3 \times 10^{44}$ & $3.9 \times 10^{22}$ \\
\hline & 2 & $-955^{\prime \prime}$ & $-10^{\prime \prime}$ & $3 \times 10^{8}$ & $8.4 \times 10^{16}$ & $5 \times 10^{9}$ & - & 8 & $2.7 \times 10^{43}$ & $9.2 \times 10^{21}$ \\
\hline & 3 & $-940^{\prime \prime}$ & $-30^{\prime \prime}$ & $5 \times 10^{8}$ & $1.8 \times 10^{17}$ & $2 \times 10^{9}$ & 22 & 13 & $1.4 \times 10^{44}$ & $4.7 \times 10^{22}$ \\
\hline & 4 & $-940^{\prime \prime}$ & $-146^{\prime \prime}$ & $5 \times 10^{8}$ & $2.2 \times 10^{17}$ & $2 \times 10^{9}$ & - & 14 & $1.4 \times 10^{44}$ & $5.5 \times 10^{22}$ \\
\hline & 5 & $932^{\prime \prime}$ & $20^{\prime \prime}$ & $4 \times 10^{8}$ & $1.6 \times 10^{17}$ & $2 \times 10^{9}$ & 15 & 12 & $3.9 \times 10^{43}$ & $1.6 \times 10^{22}$ \\
\hline & 6 & $930^{\prime \prime}$ & $-60^{\prime \prime}$ & $5 \times 10^{8}$ & $1.6 \times 10^{17}$ & $1 \times 10^{9}$ & 11 & 13 & $8.1 \times 10^{43}$ & $2.8 \times 10^{22}$ \\
\hline & 7 & $10^{\prime \prime}$ & $735^{\prime \prime}$ & $5 \times 10^{8}$ & $1.7 \times 10^{17}$ & $4 \times 10^{9}$ & 13 & 11 & $5.3 \times 10^{43}$ & $1.8 \times 10^{22}$ \\
\hline \multirow{3}{*}{$\mathrm{CH}$} & 1 & $-12^{\prime \prime}$ & $820^{\prime \prime}$ & $4 \times 10^{8}$ & $9.2 \times 10^{16}$ & $4 \times 10^{9}$ & 15 & 8 & $2.3 \times 10^{43}$ & $7.6 \times 10^{21}$ \\
\hline & 2 & $10^{\prime \prime}$ & $910^{\prime \prime}$ & $5 \times 10^{8}$ & $2.3 \times 10^{17}$ & $2 \times 10^{9}$ & - & 15 & $1.2 \times 10^{44}$ & $4.4 \times 10^{22}$ \\
\hline & 3 & $0^{\prime \prime}$ & $-940^{\prime \prime}$ & $3 \times 10^{8}$ & $9.7 \times 10^{16}$ & $3 \times 10^{9}$ & 8 & 9 & $4.9 \times 10^{42}$ & $1.9 \times 10^{21}$ \\
\hline
\end{tabular}

In Table 2, the first columns present heliocentric coordinates of the bright points, which were mostly observed close to the limb. An apparent diameter of microflare is determined by using original XRT images without pixel summing. This is because the resolution of a summed pixel is not sufficient for measuring apparent size. A typical apparent diameter of the microflare is 3$5 \times 10^{8} \mathrm{~cm}$. The apparent areas of the microflares correspond to the areas covered by pixels in the XRT Al_poly images brighter than the $3 \sigma$ level.

The bottom panel of Fig. 1 shows a time series of a bright point in a coronal hole near the North limb $(\mathrm{CH} 1)$. Increased emission is detected at the center of all images at 15:48 UTC, which is similar to the quiet region event, QR1. A major difference is that no coronal dimming is found in the surroundings. Instead, a diffuse jet is seen above the microflare in the XRT Al_poly difference image. The diffuse jet extended to 50" at 15:58 UTC. The difference images indicate darkenings only in the bright point, indicating a small emission decrease from the pre-flare structure.

\subsection{Lightcurves}

Figure 3 presents lightcurves of the bright point QR1. The X-ray lightcurves are deduced from $4^{\prime \prime} \times 2^{\prime \prime}$ area, which match the resolution of the EIS data. Shortly after the Hinode observations start, a rapid rise in emission the main peak started at 14:08 UTC. The maximum flux is attained in the Be_thin and Al_poly filters at 14:10 UTC. Fluxes decrease to their pre-event level by 14:25 UTC, hence the duration of the enhanced radiance was $25 \mathrm{~min}$.

Co-spatial lightcurves of emission lines observed by EIS are also plotted in Fig. 3. Note that the temporal resolution of EIS is 6 min. An enhanced emission was observed in all emission lines at 14:10 UTC, almost in coincidence with the X-ray peak. A sharp rise in Fe $\mathrm{xv}$ reflects the fact that the Fe Xv emission is normally absent in quiet regions and is significant only during the event. The peak is also observed in other emission lines, though its enhancement is less pronounced than the Fexv. This multi-temperature peak is regarded as a heating from the chromosphere to the corona during the microflare. After the X-ray peak, another emission peak was detected in $\mathrm{He}$ II and $\mathrm{O} v$ at 14:22 UTC, while the Fe XV and Fe XII emission remained at preevent levels. The delayed peak in cool emission is interpreted as cooling of the hot plasma produced by the microflare. This hypothesis is examined in Sect. 4.7. The delay time is defined as the time between the X-ray peak and the following peak in cool emission lines, either $\mathrm{O} v$ or He II. For the QR1, the delay time was $12 \mathrm{~min}$. The hottest recorded emission was in the Fe xv line,
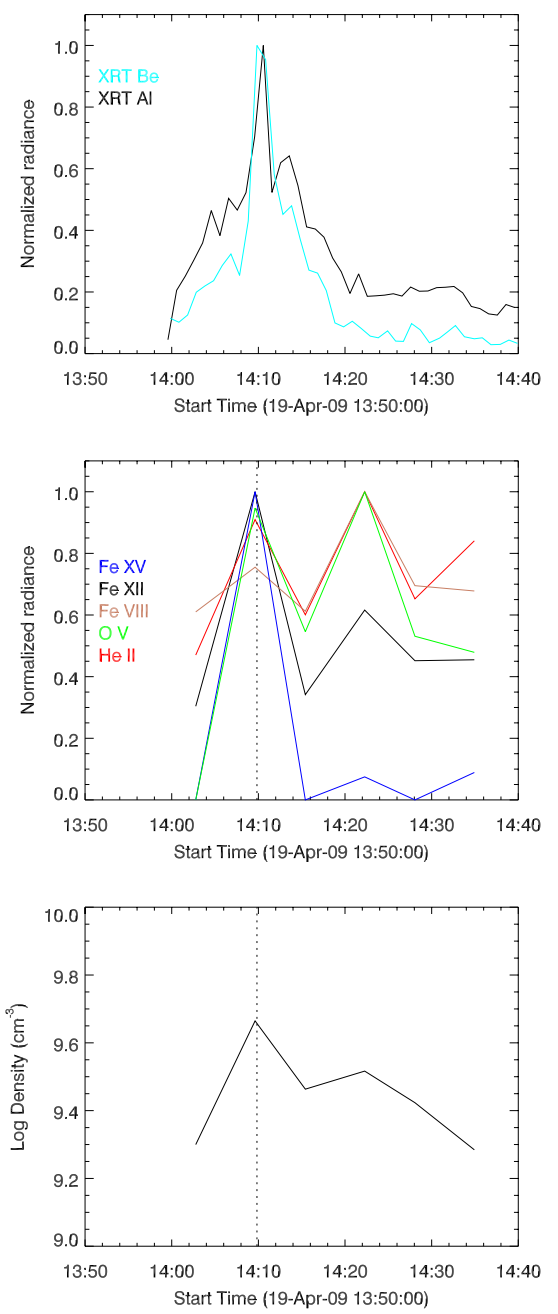

Fig. 3. Normalized lightcurves of the microflare in a quiet region (QR1). Top: X-ray lightcurves from XRT Be_thin and Al_poly filters. Middle: lightcurves from EIS emission lines. Bottom: the electron density $\left(\mathrm{cm}^{-3}\right)$ deduced from the emission ratio of FexII $\lambda 18.688 \mathrm{~nm} / \lambda 19.512 \mathrm{~nm}$.

and no emission was detected in Fe XVII. This can used to set the upper limit to the temperature of the microflare $\left(T_{\mathrm{e}}<4 \times 10^{6} \mathrm{~K}\right)$.

Figure 4 shows the lightcurves for the bright point in the coronal hole (CH1). The X-ray fluxes indicate two subpeaks; the first subpeak at 15:49 UTC and the second is at 15:53 UTC. An EIS exposure coincided with the first subpeak, which underwent 

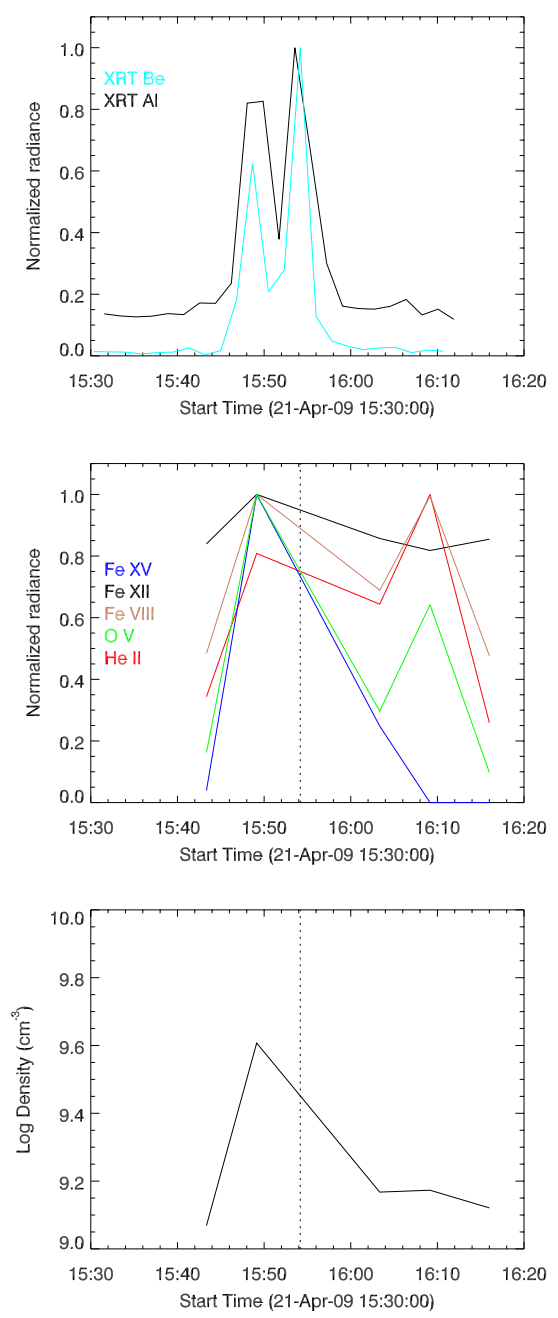

Fig. 4. The same as Fig. 3 but for the microflare in a coronal hole (CH1).

enhanced emissions in all emission lines. The following exposure missed the second peak and captured its decay phase. As the Fe xII emission gradually decreased, delayed enhancements were found in the $\mathrm{Ov}$ and He II lines. The delay between the largest X-ray peak and the cool emissions was 15 min.

It must be noted that the accuracy of the delay times can only be determined to within the EIS temporal resolution of 6 min. Nevertheless, it is worthwhile to check if the delayed cool emissions are common phenomena. Figure 5 presents lightcurves of all events for selected emissions. Delay times are measured for other events and summarized in Table 2. Delayed cool emission were found for 7 out of the 10 microflare events. No delay time is determined for QR2, QR4, and $\mathrm{CH} 2$, since no clear increase in emission was observed in the cool lines.

\subsection{Spectra of microflares}

Figure 6 presents spectra obtained by EIS coinciding with the peak of the microflare labelled QR1 at 14:10 UTC. Normalized profiles are plotted in Fig. 7. The dotted line show a spectral profile in a quiet region $10^{\prime \prime}$ apart from the microflare, except for Fe xv, for which no Fe xv emission was detected in the surrounding. The He II line indicated a redshift of $40 \mathrm{~km} \mathrm{~s}^{-1}$. The Fe vIII profile at the microflare was broader than in the quiet region, indicating that turbulent motions exist in the microflare.
The Fe XII emission increased at the microflare, and a blueshift of about $10 \mathrm{~km} \mathrm{~s}^{-1}$ was found. The Fe Xv spectrum shows its emission only at the microflare. It suggests that the hot component is confined to the microflare as the emission is absent in the surroundings. The Fe Xv spectrum shows a noticeable enhancement of the blue wing up to $-200 \mathrm{~km} \mathrm{~s}^{-1}$. The blue wing enhancements are attributed to a horizontal flow rather than a radial flow, as the radial component has a smaller contribution to the line of sight velocity due to the location of the bright point on the limb.

Spectra from the microflare $\mathrm{CH} 1$ are displayed in Figs. 8 and 9. A broadening is found in the Fe viII line. The extended wings suggest that turbulent motions exist in the microflare. Fe xv exhibits a noisy pattern because of the low count rate. However, it amounts to a significant emission after integrating over the wavelength range (Fig. 4).

Generally the emission in coronal holes is weaker than in quiet region. Extended wings of the profiles, which suggest turbulent motions in microflares, are observed in both quiet region and coronal hole.

\subsection{Electron densities}

The electron density $n_{\mathrm{e}}$ in the microflare is deduced from the emission ratio of Fe XII $\lambda 18.688 \mathrm{~nm}$ and $\lambda 19.512 \mathrm{~nm}$, and $n_{\mathrm{e}}$ is derived from the lightcurves of the two emission lines, which are processed in the same way as in Sect. 4.2. The line ratio analysis is used because the $n_{\mathrm{e}}$ can be deduced without any assumptions about the volume or filling factor of the plasma. The CHIANTI atomic database version 6.01 (Dere et al. 2009, 1997) estimates that the ratio of the Fe XII emission lines varies from $1.1 \times 10^{-2}$ to 1.7 with an electron density range of $10^{8}-10^{12} \mathrm{~cm}^{-3}$. Electron densities derived from the Fe XII ratio are plotted in the bottom panels of Figs. 3 and 4.

The density of QR1 before the microflare was $2 \times 10^{9} \mathrm{~cm}^{-3}$ (Fig. 3). It reached $4 \times 10^{9} \mathrm{~cm}^{-3}$ at the time of the peak emission in Fe XII. The uncertainty of the density estimated from the radiance measurement error is less than $20 \%$, hence the density increase is significant. The density gradually decreased to the preevent value by 14:37 UTC when the enhanced emission diminished. Before the microflare $\mathrm{CH} 1$, the density was $1 \times 10^{9} \mathrm{~cm}^{-3}$, and attained $4 \times 10^{9} \mathrm{~cm}^{-3}$ at the time of peak emission (Fig. 4). The density dropped back to $1 \times 10^{9} \mathrm{~cm}^{-3}$ when the following exposure was taken. Dere (2008) reported a typical density of $4 \times 10^{9} \mathrm{~cm}^{-3}$ in bright points, a value which falls within the QR1 density range, however, the temporal variation of the density was not studied by Dere (2008). Our study shows density enhancements associated with microflares. Both QR1 and $\mathrm{CH} 1$ indicate that the density increases reaching a peak which coincides with the peak of the Fe XII emission. The values of $n_{\mathrm{e}}$ derived at the Fe xII peak emission of the other microflares identified are presented in Table 2.

Shimizu (1995) carried out statistical study of microflares in an active region and derived a typical density of $2 \times 10^{9} \mathrm{~cm}^{-3}-$ $2 \times 10^{10} \mathrm{~cm}^{-3}$. Although our dataset is not sufficient for a statistical study, derived densities are below $5 \times 10^{9} \mathrm{~cm}^{-3}$, hence the densities of microflares in quiet region and coronal holes tend to be lower than those in active regions.

\subsection{Emission measure of microflares}

The temperature distribution of the microflare is studied by using emission lines over a wide temperature range. A contribution 

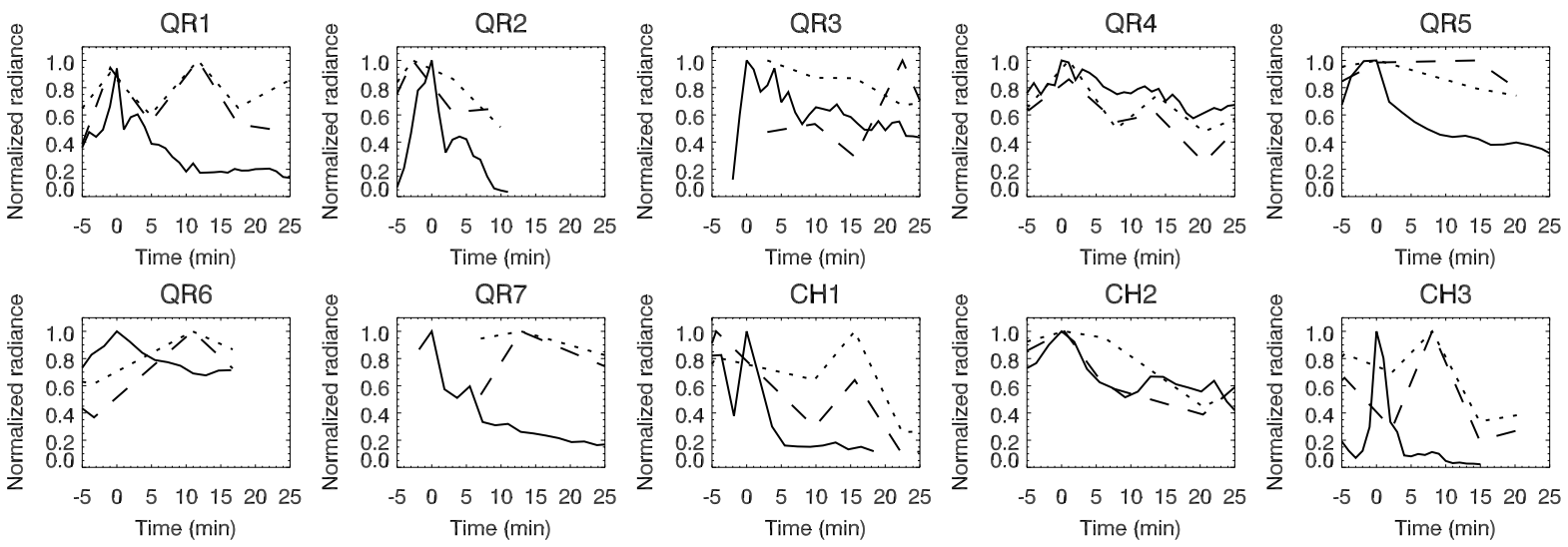

Fig. 5. Normalized lightcurves of XRT Al_poly (solid line), He II (dotted line), and Ov (dashed line) for all microflares. Horizontal axis indicates the time relative to the XRT Al_poly peak.

function of each emission line is calculated using the CHIANTI atomic database and applying coronal abundance determined by Feldman et al. (1992). The column emission measure (EM) is derived by the equation

$E M=I / g\left(T, n_{\mathrm{e}}\right)$

where $I$ is the observed radiance. and $g\left(T, n_{\mathrm{e}}\right)$ denotes the contribution function of the emission line. Figures 10 and 11 present loci plots of the microflares. Each curve presents a column emission measure assuming that the measured emission originates from an isothermal plasma at a given temperature. If the emitting plasma is purely isothermal, all the curves should meet at one temperature, which is not the case here. Convergence of multiple curves around $1 \times 10^{6} \mathrm{~K}$ implies that the emission measure peaks at that temperature. Warren \& Brooks (2009) pointed out that the emission measure is strongly peaked at $1 \times 10^{6} \mathrm{~K}$ in quiet regions, which is consistent with our results. The Fe Xv curve reaches higher than the curves of Fe vIII Fe XI, and Fe XII at $1 \times 10^{6} \mathrm{~K}$, indicating an additional high temperature component around $2 \times 10^{6} \mathrm{~K}$ in the microflare. A sporadic emission detected with XRT Be_thin filters in Fig. 1 also supports this scenario. The fact that no emission was detected in Fe XVII suggests the highest temperature is below $4 \times 10^{6} \mathrm{~K}$. Differential emission measure (DEM) analysis would give us more detailed information, however, a DEM analysis was not possible with our data set because of the small number of emission lines available.

\subsection{Radiative loss rate}

Assuming an isothermal plasma, the radiative loss from microflares is estimated by using a total radiative loss function. Since the emission measure of the microflare peaks around $1 \times 10^{6} \mathrm{~K}$ as discussed above, the column emission measure at that temperature is derived from Eq. (1) by using the Fe XII $\lambda 19.512 \mathrm{~nm}$ radiance at the peak time of the lightcurves. The column emission measure is multiplied by the apparent area of the microflare to obtain a total emission measure. A radiative loss function is derived from the CHIANTI atomic database assuming a typical density of $3 \times 10^{9} \mathrm{~cm}^{-3}$ from Table 2 . The radiative loss function is estimated to be $4 \times 10^{-22} \mathrm{erg} \mathrm{cm}^{3} \mathrm{~s}^{-1}$ at the temperature of $1 \times 10^{6} \mathrm{~K}$. Radiative loss rates of microflares are calculated by multiplying the radiative loss function and the total emission measure, which are listed in the last column of Table 2. The radiative loss rates range from $2 \times 10^{21}$ to $6 \times 10^{22} \mathrm{erg} \mathrm{s}^{-1}$.

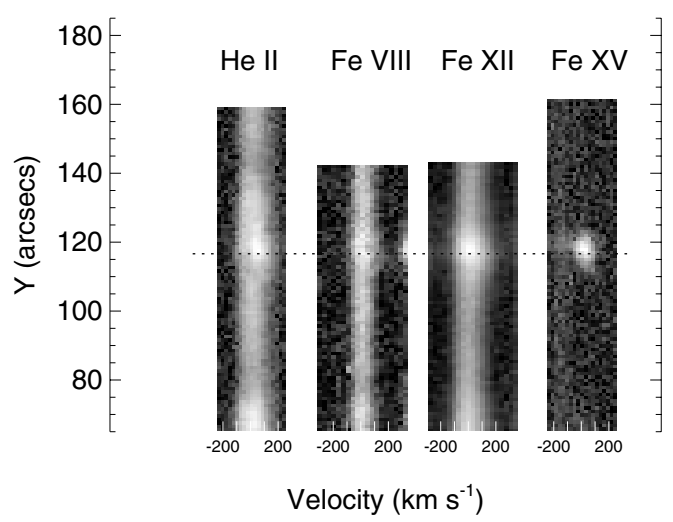

Fig. 6. Spectra in He II $\lambda 25.632 \mathrm{~nm}$, Fe vIII $\lambda 18.660 \mathrm{~nm}$, Fe XII $\lambda 19.512 \mathrm{~nm}$, and Fe xv $\lambda 28.416 \mathrm{~nm}$ recorded across the microflare QR1 at its peak emission time. The horizontal axis shows Doppler velocity relative to the line center position. Dashed line marks the location of the microflare.

As shown in Figs. 10 and 11, actual microflares are not isothermal. Therefore, our results are considered as the lower limit of the radiative loss rate since the remaining component could also contribute to the radiative loss.

\subsection{Estimated cooling times}

The observed delay times between the peaks in X-ray and cool emissions are in the 8-21 min range (Table 2). Theoretical cooling times are computed to determine if the delay times can be attributed to the cooling of hot coronal loops. Cargill et al. (1995) derived an analytical solution for the cooling time from a combination of conductive cooling and radiative cooling, taking into account chromospheric evaporation. It requires temperature $T_{0}$, density $n_{\mathrm{e}}$, and loop length $L$ as initial parameters (from Equation 14E in Cargill et al. 1995).

$\tau=2.35 \times 10^{-2} \frac{L^{5 / 6}}{T_{0}^{1 / 6} n_{\mathrm{e}}^{1 / 6}}$.

Although the internal structure of microflares is not resolved in our observations, the loop size is assumed to be an arc of an apparent diameter of the microflare. The density is estimated from 
S. Kamio et al.: Evolution of microflares associated with bright points in coronal holes and in quiet regions
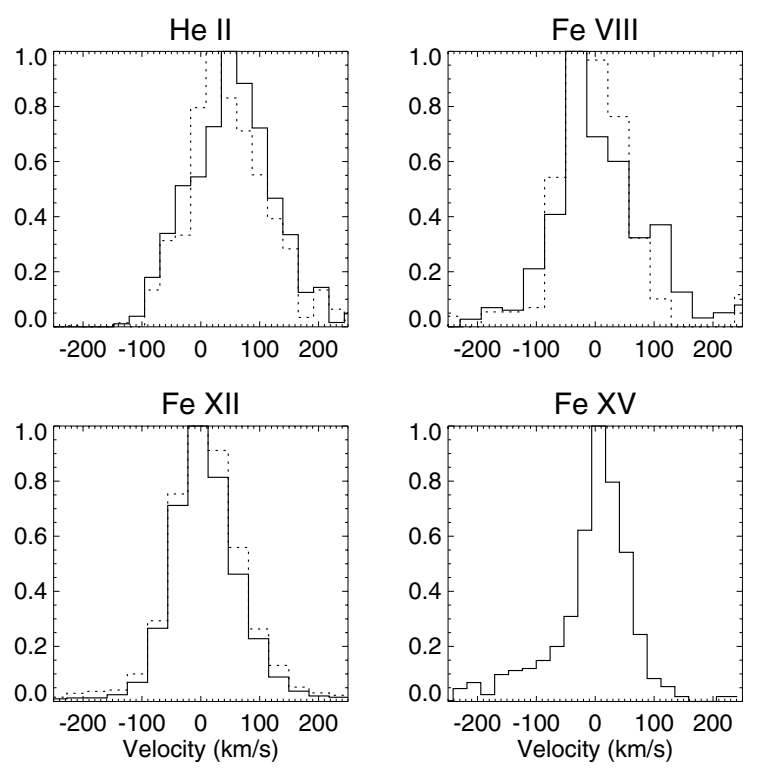

Fig. 7. Normalized spectral profiles at the microflare QR1 marked by dashed line in Fig. 6. The profiles are normalized by the peak emission. The dotted lines indicate profiles in a quiet region neighboring the microflare.

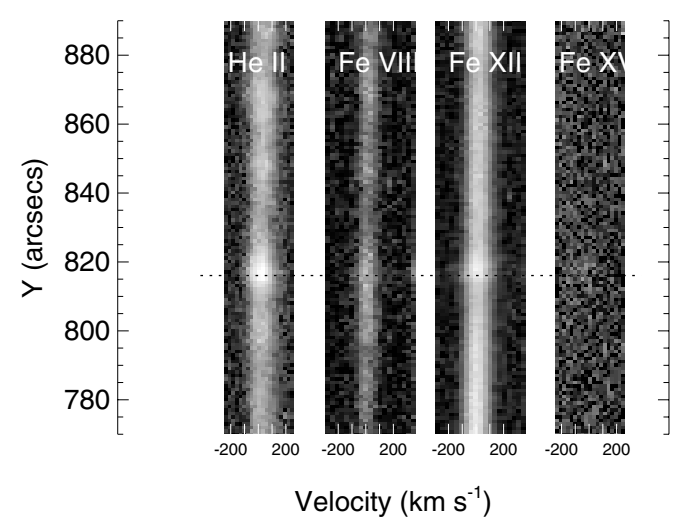

Fig. 8. The same as Fig. 6 but for the microflare CH1.

the Fe xII emission ratio analysis in the previous section. The initial temperature is set to $2 \times 10^{6} \mathrm{~K}$, the temperature of maximum fractional ionization for Fexv. The cooling time is calculated down to $10^{5} \mathrm{~K}$. The estimated cooling times are presented in Table 2, which is 9 min for QR1 and 8 min for $\mathrm{CH} 1$.

Judging from the lightcurves, the observed time delay between the peaks in the X-ray and cool emission lines is $12 \mathrm{~min}$ for QR1 and $15 \mathrm{~min}$ for CH1 (Figs. 3 and 4). This discrepancy between estimated cooling time and observed delay times is comparable to the crude temporal resolution of the rastering spectrometers, which may have missed the real emission peak. The uncertainty of the loop length estimation could cause error in the cooling time calculation, since the loop was not resolved in XRT images. If the actual loop was longer than our estimation, the cooling time could be longer as it largely depends on $L$ (Eq. (2)). As an alternative, the coincidence of independent brightenings in the transition region could be an explanation. But considering the fact that the delayed cool emissions are observed in 7 out of 10 events (Fig. 5), the cool emissions are likely to be
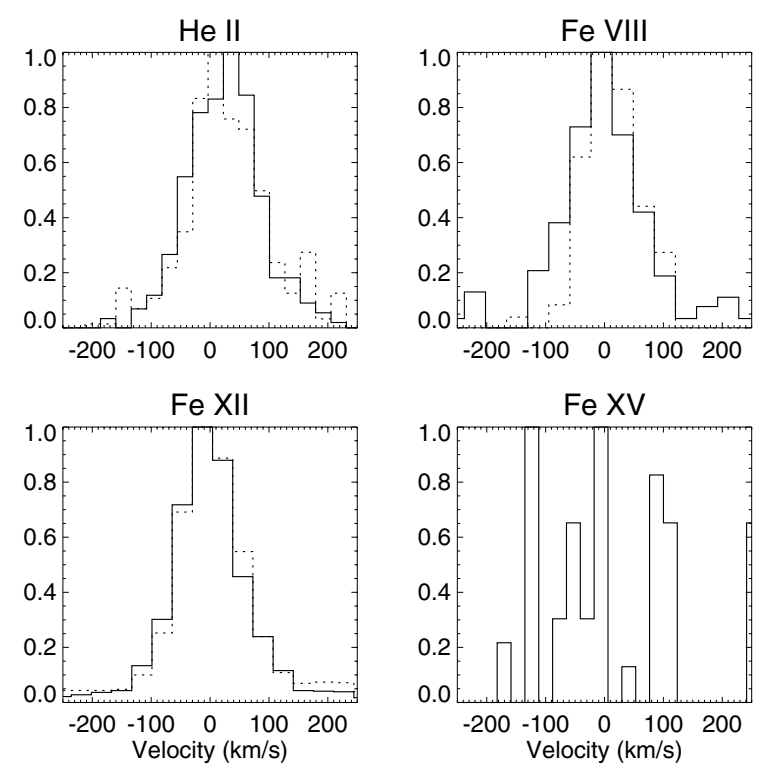

Fig. 9. Normalized spectral profiles of the microflare $\mathrm{CH} 1$ indicated with dashed line in Fig. 8.

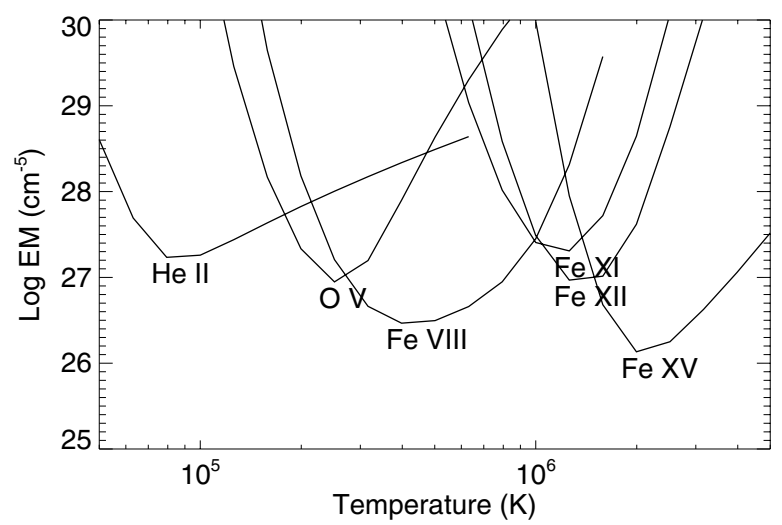

Fig. 10. Loci plot derived from emissions within the microflare QR1.

related to microflares, rather than coincidence of other brightening events in transition region. Another possibility is that the simplified loop cooling model might be inadequate to reproduce the observations.

The estimated cooling times of microflares are much shorter than those of large scale flares. This is explained by the short conductive cooling time scale of small coronal loops expressed as $\tau_{\text {cond }}=4 \times 10^{-10} n_{\mathrm{e}} L^{2} T_{0}^{-5 / 2}$ where $n_{\mathrm{e}}, L$, and $T_{0}$ respectively denote density, loop length, and temperature (Cargill et al. 1995). Shorter loop lengths result in shorter conductive cooling time, while radiative cooling does not depend on the loop length.

\section{Discussion}

The temporal evolution of microflares is traced in quiet regions and in polar coronal holes. The lightcurves exhibit impulsive increases in emission over a wide temperature ranging from He II to $\mathrm{Fe} x \mathrm{x}$. Hot emission peaks in X-ray, $\mathrm{Fexv}$, and Fe xII were followed by enhancements in cool emission in $\mathrm{He}$ II or in $\mathrm{O} v$ for 7 out of 10 microflares. The delay time between the peaks in X-ray and cool emission was 8-22 min. Such delayed peaks 


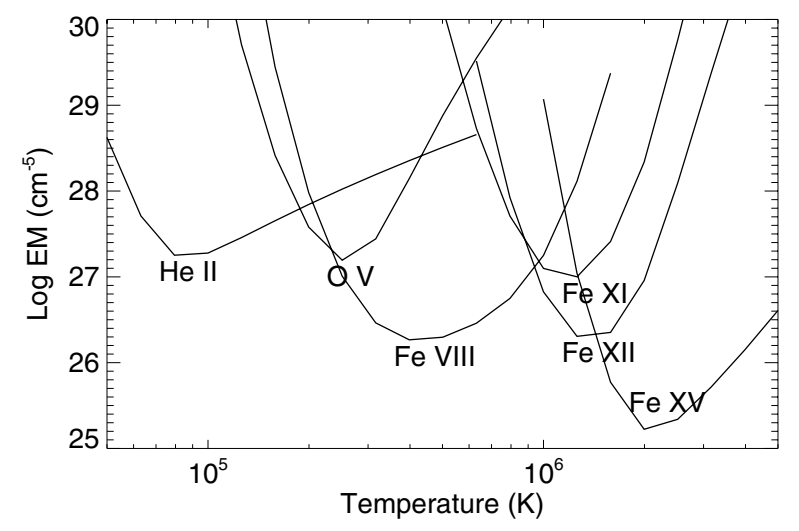

Fig. 11. Loci plot derived from emissions within the microflare $\mathrm{CH} 1$.

in cool emission are common to large flares (Curdt et al. 2004; Teriaca et al. 2006; Raftery et al. 2009), although the time delays vary from tens of minutes to hours. The lightcurve behavior is interpreted as the cooling of hot plasma produced by impulsive heating at the beginning of the flare. To test this hypothesis, observed time delays are compared with the simple loop cooling model by Cargill et al. (1995). Although the temporal resolution is not better than $6 \mathrm{~min}$, theoretical cooling times generally agree with observations. Therefore, lightcurves of microflares can be explained as impulsive heating followed by the cooling of small coronal loops. The small loop length of microflare compared to that of active regions results in a shorter conductive cooling time, while the radiative cooling time is independent of the loop length. The short time scale of microflares is largely due to the small length of the loops.

Benz \& Krucker (1999) investigated the cross-correlations between lightcurves of different emission lines and claimed that $\mathrm{He} \mathrm{I}$ and $\mathrm{O} v$ peak emission precedes the Fe XII peak emission by $5 \mathrm{~min}$, although the correlation coefficients were low. They infer that the chromosphere is heated to coronal temperatures by small heating events. We did not find noticeable peaks in cool emissions prior to the coronal emission peak. A possible reason is that the temporal resolution of our observations was insufficient to resolve rapidly changing phenomena. Nevertheless, enhanced cool emission was found at the time of peak X-ray flux, which could be a signature of chromospheric evaporation analogous to large flares. Benz \& Krucker (1999) also reported that the Fe Ix/x lightcurve lags the Fe xII lightcurve by 23 s, which was interpreted as coronal plasma cooling.

It is interesting to note that lightcurves of microflares in quiet regions and in coronal holes have common characteristics. Delayed cool emission in a bright point associated with a polar coronal jet was reported (Culhane et al. 2007b). In our study, the delayed emission was found both in coronal holes and in quiet regions. It suggests that the cooling of microflares in coronal hole and in quiet region are explained by the same mechanism.

The line broadening of spectra recorded during microflares can be interpreted as superposition of unresolved flows of up to $-200 \mathrm{~km} \mathrm{~s}^{-1}$. Mariska et al. (2007) found an impulsive intensity increase accompanied by blueshifts in Fe XII and Fe XV in an active region loop. An impulsive heating could produce a flow along the loop. The flow would be observed as a line broadening rather than a distinct flow if coronal structures are smaller than resolution of the instrument. Berkebile-Stoiser et al. (2009) detected upflows and downflows of $10-40 \mathrm{~km} \mathrm{~s}^{-1}$ in He I, O v, and $\mathrm{Ne}$ vi for different microflares near disk center. But no distinct flows were found from our observations in $\mathrm{He}$ II or O v. A possible reason is that the flows were almost vertical in the transition region and thus not detected near the limb in our observations. The broadened spectra of Fe $\mathrm{xv}$ are interpreted as flows along the coronal loop (Fig. 7).

We found increases in density accompanying the increase in emission, which supports chromospheric evaporation models. Increased coronal densities have been found in previous studies with EIS. Chifor et al. (2008) showed a density dependence on the upflow velocity in a coronal jet. Watanabe et al. (2009) derived a high density of $\geq 10^{11} \mathrm{~cm}^{-3}$ in a flare loop from Fe XIII line ratio analysis. Our study showed the temporal variation of the coronal density associated with microflares. It implies that impulsive heating led to the chromospheric evaporation. The evaporation flow along the coronal loop could contribute to the line broadenings mentioned above.

Although the properties of microflares are quite similar in quiet regions and in coronal holes, a notable difference is found in their surroundings. Microflares in quiet regions are accompanied by coronal dimmings, while microflares in coronal holes are associated with jets. This is probably due to a difference in the magnetic field configuration of the surroundings. Magnetic loops in bright points interact with open fields in coronal holes to produce coronal jets (Shibata et al. 1994). The hot plasma ejected along the open fields caused a diffuse jet above the bright point in Fig. 1. Pariat et al. (2009) performed 3D MHD simulations of a twisted emerging flux rope reconnecting with open magnetic fields. They claimed that the reconnection underwent two distinctive regimes: an impulsive mode and a quasi-steady mode (Pariat et al. 2010). We speculate that the impulsive reconnection produces microflares, while the quasi-steady reconnection maintained persistent emission in the coronal bright point. In our observations, no clear signature of a jet is seen in the spectra. It is probably due to a faint emission from the jet and short exposure time of our observations. Although the jet is noticeable in difference images in Fig. 1, a dominant emission comes from a background corona, hence the spectrum did not indicate a significant Doppler shift as a whole.

The microflares and associated coronal dimmings in quiet regions are essentially the same as those observed in active regions (Innes et al. 2009, 2010). Quiet regions are supposedly filled with closed magnetic fields (Ito et al. 2010). In the case of bright point QR1, the coronal dimming started in the surroundings when the emission began to rise in the bright point. The changes in magnetic field configuration which were initiated by reconnection are likely to be the cause of coronal dimmings in the surroundings. For large scale flares, it has been established that coronal dimmings are associated with CMEs by statistical studies (Bewsher et al. 2008; Reinard \& Biesecker 2008). A possible explanation for the coronal dimming is the removal of overlying coronal field (Innes et al. 2010). Archontis \& Hood (2010) used 3D MHD simulations to study flux emergence into horizontal fields. They demonstrated pre-existing magnetic fields in the corona are peeled off via reconnections with the emerging flux. We speculate that coronal magnetic fields are removed through reconnections at microflares to form the coronal dimmings. In our dataset no clear signature of eruption is detected. A possible reason is that it is not easy to identify eruptions in He II $30.4 \mathrm{~nm}$ on-disk images, because the background emission is highly structured and dynamic. In addition, the 10 min cadence of our STEREO dataset might not be sufficient to detect shortlived eruptions. Microflares are often accompanied by cool eruptions. Kamio et al. (2010a) reported an X-ray jet associated with a cool eruption in He II $30.4 \mathrm{~nm}$ above the limb. Further observa- 
tions with higher cadence are needed to establish a relationship between microflares and cool eruptions.

We focused on microflares in quiet regions and coronal holes, but microflares in active regions indicate higher temperatures. In our study, the hottest emission line in microflares is $\mathrm{Fe} x \mathrm{x}$ at $2 \times 10^{6} \mathrm{~K}$. Shimizu (1995) obtained a temperature range of $4 \times 10^{6} \sim 8 \times 10^{6} \mathrm{~K}$ from a statistical study of microflares in active regions. Brosius \& Holman (2009) detected Fe XIX emission $\left(8 \times 10^{6} \mathrm{~K}\right)$ in a microflare in an active region. Scaling laws of the flare peak temperature and emission measure have been determined (Feldman et al. 1996; Shibata \& Yokoyama 1999), implying that different scales of flares are produced by the same mechanism. If the relationship could be extended to a lower emission measure, small events are expected to show a low temperature. Aschwanden et al. (2008) derived a scaling law of $E M \propto T^{4.7}$ from microflares to large scale flares. Although the number of events detected in our study is limited, their scaling law can be compared with our results at $1 \times 10^{6} \mathrm{~K}$. The scaling law by Aschwanden et al. (2008) estimates an emission measure of $10^{44} \mathrm{~cm}^{-3}$ at $1 \times 10^{6} \mathrm{~K}$, which is close to $10^{42} \sim 10^{44} \mathrm{~cm}^{-3}$ from our results. Probably improved resolution and sensitivity of instruments used allowed us to detected small events in quiet regions and in coronal holes. Although we selected bright points which were obvious in the X-ray images, cooler events which would not show up in X-ray were also reported. Young et al. (2007) reported an active region brightening which was visible up to Fe xII. Therefore, transient brightenings with a lower temperature than our study have been shown to exist.

\section{Conclusions}

We traced the evolution of microflares in coronal bright points. The lightcurves of microflares show a common behavior in quiet regions and in coronal holes, starting with a impulsive rise in a wide temperature range up to $\mathrm{Fe} x \mathrm{xv}$ at $2 \times 10^{6} \mathrm{~K}$. The spectra at the impulsive peak exhibited broad profiles, which are interpreted as a superposition of flows caused by the microflares. After the hot coronal emissions decreased to normal level, enhanced emissions were noticed in cool emissions with a delay of 8-22 min. The observed delay times agree with a simple cooling model of a coronal loop (Cargill et al. 1995), though the temporal resolution of the measurement is not high enough. The line ratio analysis indicated a density increase coinciding with an impulsive emission peak, supporting chromospheric evaporation models.

These characteristics of microflares are common to active region flares, which suggests that microflares and large flares are produced by the same mechanism. The empirical flare scaling law (Aschwanden et al. 2008) falls in the range of the observed emission measure and temperature of microflares. A notable difference is found in the surroundings of microflares; diffuse coronal jets are produced above bright point in coronal holes while coronal dimmings are formed in quiet regions. They are thought to be due to differences in the magnetic field configuration of the surroundings of the microflares. An interaction of an emerging flux with open fields and closed fields must be examined in detail by theoretical studies.

Acknowledgements. We would like to thank the referee for constructive suggestions. Hinode is a Japanese mission developed and launched by ISAS/JAXA, with NAOJ as domestic partner and NASA and STFC (UK) as international partners. It is operated by these agencies in co-operation with ESA and NSC (Norway). STEREO is a project of NASA. The data from the SECCHI instrument used here was produced by an international consortion led by the Naval Research Lab (USA). The STEREO contributions by the MPS were supported by DLR grant $500 C 0501$.

\section{References}

Archontis, V., \& Hood, A. W. 2010, A\&A, 514, A56

Aschwanden, M. J., Stern, R. A., \& Güdel, M. 2008, ApJ, 672, 659

Benz, A. O., \& Krucker, S. 1999, A\&A, 341, 286

Berkebile-Stoiser, S., Gömöry, P., Veronig, A. M., Rybák, J., \& Sütterlin, P. 2009, A\&A, 505, 811

Bewsher, D., Harrison, R. A., \& Brown, D. S. 2008, A\&A, 478, 897

Brosius, J. W., \& Holman, G. D. 2009, ApJ, 692, 492

Brown, C. M., Feldman, U., Seely, J. F., Korendyke, C. M., \& Hara, H. 2008, ApJS, 176, 511

Cargill, P. J., Mariska, J. T., \& Antiochos, S. K. 1995, ApJ, 439, 1034

Carmichael, H. 1964, in The Physics of Solar Flares, 451

Chifor, C., Young, P. R., Isobe, H., et al. 2008, A\&A, 481, L57

Cirtain, J. W., Golub, L., Lundquist, L., et al. 2007, Science, 318, 1580

Culhane, J. L., Harra, L. K., James, A. M., et al. 2007a, Sol. Phys., 243, 19

Culhane, L., Harra, L. K., Baker, D., et al. 2007b, PASJ, 59, S751

Curdt, W., Wang, T. J., Dwivedi, B. N., Kliem, B., \& Dammasch, I. E. 2004, in SOHO 13 Waves, Oscillations and Small-Scale Transients Events in the Solar Atmosphere: Joint View from SOHO and TRACE, ed. H. Lacoste, ESA-SP, 547,333

Dere, K. P. 2008, A\&A, 491, 561

Dere, K. P., Landi, E., Mason, H. E., Monsignori Fossi, B. C., \& Young, P. R. 1997, A\&AS, 125, 149

Dere, K. P., Landi, E., Young, P. R., et al. 2009, A\&A, 498, 915

Feldman, U., Mandelbaum, P., Seely, J. F., Doschek, G. A., \& Gursky, H. 1992, ApJS, 81, 387

Feldman, U., Doschek, G. A., Behring, W. E., \& Phillips, K. J. H. 1996, ApJ, 460, 1034

Freeland, S. L., \& Handy, B. N. 1998, Sol. Phys., 182, 497

Golub, L., Krieger, A. S., Silk, J. K., Timothy, A. F., \& Vaiana, G. S. 1974, ApJ, 189, L93

Golub, L., Krieger, A. S., Harvey, J. W., \& Vaiana, G. S. 1977, Sol. Phys., 53, 111

Golub, L., Deluca, E., Austin, G., et al. 2007, Sol. Phys., 243, 63

Hirayama, T. 1974, Sol. Phys., 34, 323

Howard, R. A., Moses, J. D., Vourlidas, A., et al. 2008, Space Sci. Rev., 136, 67

Innes, D. E., Genetelli, A., Attie, R., \& Potts, H. E. 2009, A\&A, 495, 319

Innes, D. E., McIntosh, S. W., \& Pietarila, A. 2010, A\&A, 517, L7

Ito, H., Tsuneta, S., Shiota, D., Tokumaru, M., \& Fujiki, K. 2010, ApJ, 719, 131

Kaiser, M. L., Kucera, T. A., Davila, J. M., et al. 2008, Space Sci. Rev., 136, 5

Kamio, S., Hara, H., Watanabe, T., \& Curdt, W. 2009, A\&A, 502, 345

Kamio, S., Curdt, W., Teriaca, L., Inhester, B., \& Solanki, S. K. 2010a, A\&A, 510, L1

Kamio, S., Hara, H., Watanabe, T., Fredvik, T., \& Hansteen, V. H. 2010b, Sol. Phys., 266, 209

Kopp, R. A., \& Pneuman, G. W. 1976, Sol. Phys., 50, 85

Kosugi, T., Matsuzaki, K., Sakao, T., et al. 2007a, Sol. Phys., 243, 3

Kotoku, J., Kano, R., Tsuneta, S., et al. 2007b, PASJ, 59, S735

Koutchmy, S., Hara, H., Suematsu, Y., \& Reardon, K. 1997, A\&A, 320, L33

Mariska, J. T., Warren, H. P., Ugarte-Urra, I., et al. 2007, PASJ, 59, S713

Pariat, E., Antiochos, S. K., \& DeVore, C. R. 2009, ApJ, 691, 61

Pariat, E., Antiochos, S. K., \& DeVore, C. R. 2010, ApJ, 714, 1762

Raftery, C. L., Gallagher, P. T., Milligan, R. O., \& Klimchuk, J. A. 2009, A\&A, 494, 1127

Reinard, A. A., \& Biesecker, D. A. 2008, ApJ, 674, 576

Savcheva, A., Cirtain, J., Deluca, E. E., et al. 2007, PASJ, 59, 771

Shibata, K., \& Yokoyama, T. 1999, ApJ, 526, L49

Shibata, K., Nitta, N., Strong, K. T., et al. 1994, ApJ, 431, L51

Shimizu, T. 1995, PASJ, 47, 251

Shimojo, M., Narukage, N., Kano, R., et al. 2007, PASJ, 59, S745

Strong, K. T., Harvey, K., Hirayama, T., et al. 1992, PASJ, 44, L161

Sturrock, P. A. 1966, Nature, 211, 695

Subramanian, S., Madjarska, M. S., \& Doyle, J. G. 2010, A\&A, 516, A50

Teriaca, L., Falchi, A., Falciani, R., Cauzzi, G., \& Maltagliati, L. 2006, A\&A, 455,1123

Thompson, B. J., Plunkett, S. P., Gurman, J. B., et al. 1998, Geophys. Res. Lett., 25,2465

Warren, H. P., \& Brooks, D. H. 2009, ApJ, 700, 762

Watanabe, T., Hara, H., Yamamoto, N., et al. 2009, ApJ, 692, 1294

Wülser, J.-P., Lemen, J. R., Tarbell, T. D., et al. 2004, in SPIE Conf. Ser. 5171, ed. S. Fineschi, \& M. A. Gummin, 111

Young, P. R., Del Zanna, G., Mason, H. E., et al. 2007, PASJ, 59, S727 